\title{
Barriers to conducting cancer trials in Canada: an analysis of key informant interviews
}

\author{
C. Bentley PhD, ${ }^{*+}$ S. Sundquist, ${ }^{\ddagger \S}$ J. Dancey MD, ${ }^{\ddagger \S \| \#}$ and S. Peacock DPhil ${ }^{*+* *}$
}

\begin{abstract}
Background In Canada, there is growing evidence that oncology clinical trials units (CTUs) and programs face serious financial challenges. Investment in cancer research in Canada has declined almost $20 \%$ in the 5 years since its peak in 2011, and the costs of conducting leading-edge trials are rising. Clinical trials units must therefore be strategic about which studies they open. We interviewed Canadian health care professionals responsible for running cancer trials programs to identify the barriers to sustainability that they face.
\end{abstract}

Methods One-on-one telephone interviews were conducted with clinicians and clinical research professionals at oncology crus in Canada. We asked for their perspectives about the barriers to conducting trials at their institutions, in their provinces, and nationwide. Interviews were digitally recorded, transcribed, anonymized, and coded in the NVivo software application (version 11: QSR International, Melbourne, Australia). The initial coding structure was informed by the interview script, with new concepts drawn out and coded during analysis, using a constant comparative approach.

Results Between June 2017 and November 2018, 25 interviews were conducted. Key barriers that participants identified were

insufficient stable funding to support trials infrastructure and retain staff;

the need to adopt strict cost-recovery policies, leading to fewer academic trials in portfolios; and

an overreliance on industry to fund clinical research in Canada.

Conclusions Funding uncertainties have led cтUs to increasingly rely on industry sponsorship and more stringent feasibility thresholds to remain solvent. Retaining skilled trials staff can create efficiencies in opening and running studies, with spillover effects of more trials being open to patients. More academic studies are needed to curb industry's influence.

Key Words Clinical trials, sustainability, clinical trials units, oncology research investment, Canada

Curr Oncol. 2020 June:27(3)e307-e312

www.current-oncology.com

\section{INTRODUCTION}

Clinical trials are essential to advancing cancer care, serving to translate promising drugs and technologies into therapies that improve the lives of people with cancer. Yet running leading-edge clinical trials programs represents a major research undertaking, leading to rising $\operatorname{costs}^{1,2}$. A recent scoping review of the international empirical literature on the costs and benefits of conducting clinical trials showed that trial expenditures are driven upward primarily by the time and resources required to activate trials and to reach accrual targets ${ }^{3}$. As trial costs continue to rise, revenue streams in North America for publicly funded trials have narrowed. In the United States, fewer funds are being directed to scientific research generally ${ }^{4}$ and to the National Cancer Institute's National Clinical Trials Network specifically ${ }^{1}$. In Canada, investment in cancer research has similarly declined, from a peak of $\$ 583$ million in 2011 to $\$ 473$ million in 2016, with public expenditures on cancer care outpacing cancer research investment ${ }^{5}$. Declines in research investment and rising trial costs mean that leaders of oncology clinical trials units (СTUs) and programs must 
strike a careful balance between fiscal viability and the capacity to advance clinical research to improve the lives of patients.

In Canada, there is further evidence that oncology trials and CTUs face serious financial challenges. In Canada's single-payer health system, clinical research itself is often perceived as a costly add-on to the standard of care ${ }^{6,7}$, and institutional cost recovery for non-standard-of-care items is on the rise ${ }^{8}$. Oncology CTUs and trials programs-which are responsible for all aspects of trial site activities, including accruing patients to trials, collecting study-related data and following patients, adhering to regulatory requirements, and sustaining the trial operations infrastructureare also under threat. Funding streams to support cTUs are unreliable because they depend in part on funding from cancer foundations, research awards in Canada's highly competitive grant environment (in 2016, approximately $\$ 50.5$ million was invested nationally in cancer clinical research, a figure that included both grant funding and government-sourced CTU infrastructure support ${ }^{5}$ ), and on per-patient accrual incentive amounts that are allocated only to top-performing centres. Many CTUs and departments at academic institutions reportedly operate at a loss ${ }^{9}$.

Fiscal uncertainty and the imperative to recover costs have led oncology CTU leaders to be strategic about which trials to open. Often those leaders turn to the pharmaceutical industry as a funding source, because industry-sponsored studies tend to reimburse direct and indirect research costs to a greater extent than do academic-sponsored studies. That shift to industry sponsorship has consequences for cancer clinical research in Canada. First, pharmaceutical companies are developing oncology drugs that are increasingly unaffordable to place on public formularies because of their high price tags ${ }^{10,11}$. In addition, often only a small patient population stands to benefit from the new drugs, and the benefits gained are incremental. It is thus left to under-resourced, non-industry-sponsored studies and trialists to pose and pursue important questions that are not directly related to industry interests, but that are relevant to patient care and to the understanding of cancer. Taken together, this set of circumstances underscores the need to enhance academic cancer clinical research in Canada so as to address the interests of Canadian patients, health systems, and the scientific community into the future.

This interview study was undertaken to understand the specific challenges to running cancer CTUs and programs from the perspectives of clinicians and clinical research professionals at the front lines of clinical research in Canada. Specifically, we sought their views to clarify systemic barriers to conducting clinical trials at their institutions, in their provinces, and nationwide, and to develop strategies for improvement.

\section{METHODS}

\section{Recruitment and Sample}

This qualitative study used data from one-on-one semi-structured interviews with clinicians and clinical research professionals working at cancer centres, hospitals, and institutions that conduct clinical research in Canada. A purposeful sampling strategy was used for recruitment, with attention paid to representation by province or region and role (that is, clinician or clinical research professional). Study information and an invitation to participate were sent to potential participants by e-mail, with 2 follow-up messages sent to nonrespondents after $2-3$ weeks. Interested participants were sent a consent form, and a telephone interview was scheduled for a mutually convenient time.

In qualitative studies, sample size is based on the number of participants needed to achieve concept saturation, defined as the point in the data collection process when little or no new relevant information emerges and collecting more data will not add new understandings to identified concepts ${ }^{12,13}$. Concept saturation depends on the research question, the study design, and the heterogeneity and number of possible participants ${ }^{14}$. Studies show that as few as 12 participants can be sufficient to explore common experiences within a group ${ }^{15}$, with 25 respondents achieving $99 \%$ saturation of concepts ${ }^{14}$.

\section{Semi-structured Interviews}

An interview script was developed, informed by a prior scoping review conducted by the research team about the costs and benefits of clinical trials and clinical trials networks ${ }^{3}$. The script contained 10 questions and was piloted $(n=2)$ and revised before implementation. Participants were asked to describe their research environment and available supports, and based on their experience, to identify the challenges or barriers to conducting clinical trials in that environment. Participants were also asked to reflect on the future of cancer trials research in Canada. Only the key or most significant barriers identified by participants are reported here.

All interviews were digitally recorded, transcribed, anonymized (for example, clinician 1, clinician 2, clinician 3 or clinical research professional 1, clinical research professional 2, clinical research professional 3) and entered into the NVivo software application (version 11: QSR International, Melbourne, Australia) before analysis. A qualitative analyst, CB, conducted all interviews and read the transcripts several times to gain a comprehensive sense of the data. CB and SP developed the approach to coding and analysis. An initial coding framework was developed based on the interview script. Following the principles of constant comparison ${ }^{16}$ and critical interpretive practice, analysis was undertaken to clarify the limits of the coding framework and to determine when additional codes were needed to capture new concepts and their contours. Axial codes were developed to map relationships between concepts. This overall approach to textual analysis adheres to the spirit of content and thematic analyses in the interpretation of the findings ${ }^{17,18}$.

Written informed consent was obtained before each interview. The University of British Columbia-British Columbia Cancer Research Ethics Board approved the study protocol (REB no. H16-00887).

\section{RESULTS}

Between June 2017 and November 2018, 25 semi-structured one-on-one telephone interviews were conducted. The long data collection period accommodated participants in 
busy clinics across several provinces (Table I). Interviews averaged 43 minutes in duration. The sample size yielded saturation of concepts relevant to the study.

All participants expressed concern over the long-term viability of cancer clinical trials programs in Canada. They identified several challenges to running oncology CTUs in Canada. Their key challenges are described in the subsections that follow.

\section{Lack of Core Funding for Clinical Trials Infrastructure}

The most commonly cited barrier to conducting cancer clinical trials in Canada was the lack of core funding to support CTU infrastructure. Almost all other barriers identified by participants flowed from that prime constraint. Overall, participants accepted that revenue streams for clinical research in Canada have narrowed, and yet they were highly critical of the lack of provincial and federal government commitment, in the form of dedicated funding, to a sustained program of oncology clinical research in Canada. Without core funding, CTUs relied on "shoestring" [clinician 6] budgets and funding from grants and foundations to retain staff and keep trials programs running.

Infrastructure for clinical trials has been a huge issue [across Canada], meaning that stable funding that's outside of per-case per-patient type funding is necessary to actually keep a research organization going, to have people with stable employment who are there. - Clinician 3

If we had provincial government support for the unit, like they do for pretty much everyone else in the cancer centre-like clinic nurses, pharmacy staff-all of those positions would receive provincial health care dollars. I think that would be one thing that would be the biggest [support] for us because we don't have the funding.

- Clinical research professional 2

Without core funding to support CTU infrastructure and staff, most units were reported to be self-financed through grants, the charitable sector, and per-case funding amounts tied to patient accrual to clinical trials.

At [our cancer centre,] we have a clinical trials unit with about 70 FTE staff. That unit is to a large extent funded by income that is brought in from clinical trials. It's not like the unit is provided by [our cancer institution] or something like that.

- Clinician 2
There's a big separation between standard of care, which is paid for by the government, and research, which needs to be paid for by not-the-government. That creates barriers, inefficiencies, and waste.

- Clinician 1

\section{CTUs Have to Be Run on a Cost-Recovery Basis}

Clinical trials units were reported to be largely selffinanced and operated on a cost-recovery basis. Participants explained that new trials had to pass institutional feasibility reviews before opening and that all trial costs were carefully itemized in contracts and tracked and invoiced by finance departments. Some participants likened running a CTU to running a "business," an analogy that garnered mixed views. Others reported the need to run some studies at a loss to maintain CTU infrastructure.

Running a trials unit is like running a small business, right? So it actually works quite well here. [Our CTU] was restructured ... by breaking it into business units, and each business unit is responsible for maintaining its own fiscal viability. You have to balance off.

- Clinician 2

I think our trial unit is very interested in breaking even, which it has to be-it is a business. But the problem is, to be a clinical research unit, the investigators need to feel like they are supported, right? And it is not about business, it's about the research.

- Clinician 5

[E]very once in a while, you almost have to take on a study that may not fully cover its costs just to make sure you continue to support all the infrastructure you have in place. You are better to take the $\$ 1,000$ the study provides even if it costs $\$ 1,500$, because at least you have $\$ 1,000$ to keep supporting your infrastructure.

- Clinician 4

At some institutions, the imperative to recover costs affected the site's capacity to conduct trials, which meant that fewer trials were open for patients and raised the prospect of reduced research capacity.

The downside of [capping the number of trials we open] is that we are far from having a clinical trial for all of the common situations where you'd want to have trials. So it's by far the exception-not the rule-to have a trial available for patients.

- Clinician 3

TABLE I Participants by role and region

\begin{tabular}{|c|c|c|c|c|c|c|c|}
\hline \multirow[t]{2}{*}{ Role } & \multirow{2}{*}{$\begin{array}{c}\text { Participants } \\
\text { (n) }\end{array}$} & \multicolumn{6}{|c|}{ Region } \\
\hline & & $\begin{array}{c}\text { Atlantic } \\
\text { Provinces }\end{array}$ & Quebec & Ontario & $\begin{array}{c}\text { Prairie } \\
\text { Provinces }\end{array}$ & $\begin{array}{l}\text { British } \\
\text { Columbia }\end{array}$ & $\begin{array}{c}\text { Pan- } \\
\text { Canadian }\end{array}$ \\
\hline Clinician & 14 & 3 & 1 & 5 & 2 & 3 & - \\
\hline Clinical research professional ${ }^{\mathrm{a}}$ & 11 & - & 2 & 2 & 4 & 2 & 1 \\
\hline
\end{tabular}

a Clinical trials administrator, manager, or coordinator; contracts officer; ethics officer. 
At some level, if you have a clinical trials department like ours, and you start saying no to everything because it doesn't meet some feasibility metric for budget, then you are only going to get smaller and smaller. Your capacity to do anything is going to get smaller.... I think a lot of institutions ... struggle, then, with how they prioritize studies that don't meet that feasibility criteria.

- Clinician 4

\section{Balancing a Trials Portfolio: Scientific Merit, Clinical Need, and Balancing the Budget}

Without core funding, and because funding from grants and cancer foundations was uncertain, CTUs relied on revenue generated from per-patient accrual allowances to be self-sustaining. Participants reported significantly higher per-case allowances from industry-sponsored trials than from academic-sponsored trials, leading CTUs to balance their books by relying on well-paying industry trials to subsidize the less remunerative academic ones. Striking the appropriate balance between industry-, academic-, and investigator-led studies in trials portfolios was an ongoing challenge. Most participants reported that their portfolios contained more industry-sponsored than academic-group studies.

Well, we have to do [industry-sponsored trials] to break even and to pay for the clinical trial people who do the trials, right? ... [W] e have to have a balance, because we use the industry trials to pay for the cooperative group or the academic trials.

- Clinician 5

The more funds you have available, the more opportunity you have to do academic trials. When the funds aren't available, then you tend to lean toward industry trials, which tend to pay more.

- Clinician 13

Although the push to be judicious about trial selection was widely shared (many saw it as encouraging стUs to make better decisions about which trials to take on), some participants felt constrained by the need to "break even."

So there's always going to be limited resources, right? But that makes us have to be selective in terms of what trials we want to do. And that's not necessarily a bad thing because it makes us really look over all the trials and say, "This is a trial that's really scientifically interesting and that we can actually accrue to."

- Clinician 7

[W]e do a fair number of industry trials. We have a group that looks at the feasibility of the trials and the scientific question. So we pick and choose.

- Clinician 8

[I] fit wasn't so much about breaking even, I would open more cooperative trials. I one hundred percent would. - Clinician 5

\section{Relying on Industry to Further Clinical Research Is Problematic}

Participants worried about Canada's reliance on industry to underwrite-and drive-clinical research to benefit Canadians, given an industry prevalence in trials portfolios. Although industry can provide new therapies to patients, it is motivated by profit and thus doesn't share the same research priorities or might not be interested in the same clinical questions as non-industry or academic trialists, according to several participants. In addition, industry is interested in quickly completing trial recruitment, focusing on highaccruing centres that consequently limit opportunities for other researchers and patients to participate in trials.

Many of the studies we participate in are unfortunately pharmacy-sponsored because you need to do a certain number of those to pay for the money-losers like [academic group trials].... But at the same time, you want your patients to have access to new treatments.... So you open studies that aren't necessarily the best question, simply to have access to a drug.

— Clinician 11

If we were only doing the industry work, all we're going to do is discover newer and more expensive drugs that have some benefit, hopefully, but it's usually marginal, and it's going to cost us more and more. If we're not supporting the academic research infrastructure, which asks questions like "Can we get away with less treatment? Can we get away with other alternatives that are not as expensive?" If we're not supporting that kind of research, we are shooting ourselves in the foot constantly.

- Clinician 2

[Most clinical trials in breast cancer] are pharma-driven and the actual endpoints on the whole do not tend to be practical endpoints that will help my patients in the clinic.

- Clinician 9

We find it's harder to get trials here because of the perception that we're small and we may not have the patient population to open that trial. So we tend to lose out on some trials that we would like to open here. — Clinician 13

Not all respondents were explicitly critical of industry collaborations, and a few viewed them as mutually beneficial.

I think industry is a hugely important part of clinical trials. They have the money, the resources. They have the drugs for interventional clinical trials, and they actually have a lot of know-how and knowledge.... So if we help industry get their drugs developed in a particular area, they will feed that drug and money back to us to do more research where we have an interest. - Clinician 1

[The industry studies we do] are all privileged earlyphase trials.... We're not doing uninteresting phase III 
or phase IV studies.... The clinical investigators are engaged. [Industry] is not just feeding us. Each clinical investigator is running a study or multiple studies and is very involved. They are champions of the studies. They're not just kind of feeding patients into a system to keep something going.

- Clinician 14

\section{Hiring and Retaining Skilled Staff Is a Challenge}

Per-case funding is a precarious revenue stream for CTUs, because it is susceptible to the ebbs and flows of the number of trials that are open at any given time and how well each trial is accruing. Many participants noted that those fluctuations in funding make it difficult to plan ahead and to hire and retain trained personnel such as clinical research associates, nurses, and recruitment specialists to open and run trials.

If you hit a dry spell where you don't enrol all the patients you thought you would, or you close highaccruing studies all at once and don't have other things open, your funds rapidly drop off while the staff pay doesn't fall. You can't lay off staff in conjunction with these fluxes, or if you do lay off staff, which sometimes happens, then you lose the mechanism to open new trials and generate that ongoing source of income. Then you open trials with more effort and take more time to open them and have reduced opportunity to recoup your cost because the trials have completed or nearly completed by the time you get your door open. - Clinician 6

Many centres ... really struggle just to secure funding to do trials. They have to really watch every nickel and dime that comes through. They have to hire temporary staff, and there are problems with that. It's hard to keep temporary staff.

— Clinical research professional 1

Moreover, relying on industry remuneration and high trial enrolment to cross-subsidize under-resourced studies can take a toll on staff.

If you are running trials with high enrolment numbers just to generate revenue so you can keep the trials unit afloat, and then you still want to do those interesting scientific studies or those types of trials, then you are asking your staff to grind really hard to do the interesting work.

- Clinical research professional 6

So if you are [doing trials] just to keep the unit running, and it's not being driven by interest and patient participation and all the rest of that, then I don't think it - I think it is really hard to keep going.

- Clinician 14

\section{DISCUSSION}

Our findings support previous work in the Canadian context that calls into question the sustainability of Canada's oncology trials programs and units ${ }^{9,19}$. Participants in our study described oncology CTUs as under-resourced and highly dependent on industry sponsorship to support trial infrastructure, provide treatment options to patients, and balance CTU budgets. They identified core funding for CTUs from provincial sources as insufficient to support CTU activity and a key barrier to developing oncology clinical research capacity at their institutions, in their provinces, and Canada-wide. Current pan-Canadian initiatives such as the Canadian Cancer Clinical Trials Network receive national- and provincial-level funding-primarily from government agencies and nongovernment organizationsto facilitate knowledge-sharing and best practices across CTUs to advance academic clinical research in Canada. However, that support is inadequate in addressing the extent of operational needs for the CTUs, which affects the availability of trial opportunities for patients and the scientific community alike, according to the participants.

Clinicians and clinical research professionals elaborated on the meso-level effect of those funding challenges, which strained human resources at trial sites, making it difficult to hire and retain skilled staff. Funding challenges also made it difficult for CTU leaders to plan ahead-that is, to estimate the number of trials to open, to determine the mix of academic and industry studies in portfolios, and to estimate staff workloads in CTUs and ancillary hospital departments carrying out trial protocols. Many participants found their work rewarding, but they were frustrated by the precarious state of funding and the constant need to hedge against budget shortfalls to keep CTUs running. Some CTUs ran trials at a loss simply to keep their infrastructure in place.

To balance their budgets, СТU leaders responded to constraints by implementing cost-recovery mechanisms, running their units "like a business," and opening more higher-paying industry-sponsored studies (compared with academic-sponsored or investigator-initiated studies). Some regarded the need to be judicious about which trials to open as elevating a trial program's scientific standards for study inclusion; for others, however, the shift to cost-recovery constrained their options of studies to open, especially at programs outside the hubs of British Columbia and Ontario, which respondents said tended to be favoured by sponsors.

Partnering with industry to advance cancer research was regarded as a practical, if worrisome, reality. For instance, opening industry-backed trials provided a strategy for giving patients access to promising new drugs. It also created learning opportunities for investigators and their staff, and allowed CTUs to cross-subsidize less-profitable studies. However, working closely with industry came with significant trade-offs. Several participants regretted what they considered to be Canada's overreliance on industry to set the agenda for clinical research in Canada. The climbing costs of new oncology drugs, the impracticability of trial endpoints, and the lack of interest in clinical questions relating to less treatment were considered markers of industry's influence in that sphere. By and large, industry-backed studies were also reported to be less scientifically interesting than those led by academic investigators, meaning that opportunities to learn from industry studies might be limited for some clinicians and CTUs. A few participants were circumspect about resource-intensive industry studies generating surplus revenue for CTUs, as has been reported elsewhere ${ }^{9}$. 
The present study has strengths and limitations. Key strengths include achieving concept saturation with the interviews conducted and acquiring participants from a variety of provinces and health care institutions across Canada. Almost half the participants (48\%) were from Ontario and British Columbia. That those two provinces are major clinical trial hubs in Canada might account for that grouping of respondents. However, as is the case with qualitative research, the participant sample is not statistically representative of all CTU professionals. Because some provinces and the territories were not represented in the sample, it is possible that experiences with different health care funding and delivery models and cTUs have not been captured.

\section{CONCLUSIONS}

In this interview study, we aimed to clarify the challenges encountered by health care professionals conducting cancer clinical research in Canada. There was broad agreement on the part of the participants that CTUs in Canada are under-resourced. Current funding mechanismsrelying extensively upon grants, the charitable sector, and per-patient accrual allowances from academic trials-were deemed inadequate and unreliable. A lack of core funding to support infrastructure needs has prompted CTUs to adopt stricter feasibility thresholds to remain solvent, with many trials portfolios tipping further in favour of more remunerative industry studies.

Several issues arise from the foregoing findings. First, the study sheds light on participant concerns about the extent of industry involvement in oncology clinical research in Canada and whether public health questions are being adequately addressed in that context. It also gives voice to the need to support academic oncology trials to ensure that those public health questions are asked to benefit Canadians and so that more trial options are open to patients. Our study also suggests that retention of skilled staff creates efficiencies at trials sites for opening and running studies. Capturing those issues provides an important opportunity to appreciate more fully the barriers to oncology clinical research encountered by health care professionals and identifies areas for systemic improvement.

\section{ACKNOWLEDGMENTS}

This study was conducted with the support of the Canadian Cancer Clinical Trials Network through financial contributions from the funders and from the Ontario Institute for Cancer Research, which receives funding from the Government of Ontario. The Canadian Centre for Applied Research in Cancer Control, which is funded by the Canadian Cancer Society (grant no. 2015-703549), also provided support.

\section{CONFLICT OF INTEREST DISCLOSURES}

We have read and understood Current Oncology's policy on disclosing conflicts of interest, and we declare the following interests: JD's and SS's institution provided funding for this study. CB and SP have no conflicts of interest to declare.

\section{AUTHOR AFFILIATIONS}

*Canadian Centre for Applied Research in Cancer Control, Vancouver, BC; 'Department of Cancer Control Research, BC Cancer, Vancouver, BC; ${ }^{\ddagger}$ Canadian Cancer Clinical Trials Network, Toronto, ON; ${ }^{\S}$ Ontario Institute for Cancer Research, Toronto, ON; IDepartment of Oncology, School of Medicine, Queens University,
Kingston, ON; \#Canadian Cancer Trials Group, Kingston, ON; **Faculty of Health Sciences, Simon Fraser University, Burnaby, BC.

\section{REFERENCES}

1. Bertagnolli MM, Canetta R, Nass SJ. Expanding publicprivate collaborations to enhance cancer drug development: a report of the Institute of Medicine's workshop series, "Implementing a National Cancer Clinical Trials System for the 21st Century." Oncologist 2014;19:1179-85.

2. Kasenda B, Schandelmaier S, Sun X, et al. on behalf of the DISCO study group. Subgroup analyses in randomised controlled trials: cohort study on trial protocols and journal publications. BMJ 2014;349:g4539.

3. Bentley C, Cressman S, van der Hoek K, Arts K, Dancey J, Peacock S. Conducting clinical trials: costs, impacts, and the value of clinical trials networks-a scoping review. Clin Trials 2019;16:183-93.

4. Moses H 3rd, Matheson DH, Cairns-Smith S, George BP, Palisch C, Dorsey ER. The anatomy of medical research: US and international comparisons. JAMA 2015;313:174-89.

5. Canadian Cancer Research Alliance (CCRA). Cancer Research Investment in Canada, 2016. Toronto, ON: Canadian Partnership Against Cancer; 2019. [Available online at: https:// www.ccra-acrc.ca/wp-content/uploads/2019/06/Investment _Report_2016_EN.pdf; cited 5 August 2019]

6. Jones B, Eliasziw M, Eigl BJ, Syme R. A comparison of incremental costs of breast cancer clinical trials to standard of care. J Clin Trials 2015;5:.

7. Jones B, Syme R, Eliasziw M, Eigl BJ. Incremental costs of prostate cancer trials: are clinical trials really a burden on a public payer system? Can Urol Assoc J 2013;7:E231-6.

8. Tang PA, Hay AE, O'Callaghan CJ, et al. Estimation of drug cost avoidance and pathology cost avoidance through participation in NCIC Clinical Trials Group phase III clinical trials in Canada. Curr Oncol 2016;23(suppl 1):S7-13.

9. Seow HY, Whelan P, Levine MN, et al. Funding oncology clinical trials: are Cooperative Group trials sustainable? JClin Oncol 2012;30:1456-61.

10. Cressman S, Browman GP, Hoch JS, Kovacic L, Peacock SJ. A time-trend economic analysis of cancer drug trials. Oncologist 2015;20:729-36.

11. Howard DH, Bach PB, Berndt ER, Conti RM. Pricing in the market for anticancer drugs. J Econ Perspect 2015;29:139-62.

12. Glaser BG, Strauss AL. The Discovery of Grounded Theory: Strategies for Qualitative Research. Chicago, IL: Aldine Publishing Company; 1967.

13. Patrick DL, Burke LB, Gwaltney CJ, et al. Content validityestablishing and reporting the evidence in newly developed patient-reported outcomes (PRO) instruments for medical product evaluation: ISPOR PRO Good Research Practices Task Force report: part 2-assessing respondent understanding. Value Health 2011;14:978-88.

14. Turner-Bowker DM, Lamoureux RE, Stokes J, et al. Informing a priori sample size estimation in qualitative concept elicitation interview studies for clinical outcome assessment instrument development. Value Health 2018;21:839-42.

15. Guest G, Bunce A, Johnson L. How many interviews are enough? An experiment with data saturation and variability. Field Methods 2006;18:59-82.

16. Strauss A, Corbin J. Basics of Qualitative Research. Thousand Oaks, CA: Sage; 1998.

17. Braun V, Clarke V. Using thematic analysis in psychology. Qual Res Psychol 2006;3:77-101.

18. Elo S, Kyngas $\mathrm{H}$. The qualitative content analysis process. JAdv Nurs 2008;62:107-15.

19. Canadian Cancer Research Alliance (CCRA). Reporton the State of Cancer Clinical Trials in Canada. Toronto, ON: CCRA; 2011. [Available online at: https://www.ccra-acrc.ca/wp-content/ uploads/2019/06/Clinical_Trials_Report_2011_EN.pdf; cited 8 August 2019] 